\title{
Weighted Fractal Networks
}

\author{
Timoteo Carletti*, Simone Righi \\ Département de Mathématique, \\ Facultés Universitaires Notre Dame de la Paix \\ 8 rempart de la vierge $B 5000$ Namur, Belgium \\ corresponding author $\left({ }^{*}\right)$ timoteo.carletti@fundp.ac.be \\ tel. +32(0)81724903 fax. +32(0)81724914
}

(Dated: November 2, 2018)

\begin{abstract}
In this paper we define a new class of weighted complex networks sharing several properties with fractal sets, and whose topology can be completely analytically characterized in terms of the involved parameters and of the fractal dimension. The proposed framework defines an unifying general theory of fractal networks able to unravel some hidden mechanisms responsible for the emergence of fractal structures in Nature.

PACS numbers: 64.60.aq Complex Networks, 89.75.Fb Structures and organization in complex systems, 89.75.Da Scale-free networks, 05.45.Df Fractals
\end{abstract}

\section{INTRODUCTION}

Complex networks have recently attracted a growing interest of scientists from different fields of research, mainly because complex networks define a powerful framework for describing, analyzing and modeling real systems that can be found in Nature and/or society. This framework allows to conjugate the micro to the macro abstraction levels: nodes can be endowed with local dynamical rules, while the whole network can be though to be composed by hierarchies of clusters of nodes, that thus exhibits aggregated behavior.

The birth of graph theory is usually attributed to L. Euler with his seminal paper concerning the "Königsberg bridge problem" (1736), but it is only in the 50's that network theory started to develop autonomously with the pioneering works of Erdős and Rényi [1]. Nowaday network theory defines a research field in its own [2, 3] and the scientific activity is mainly devoted to construct and characterize complex networks exhibiting some of the remarkable properties of real networks, scale-free [4], small-world [5], communities [6], just to mention few of them.

In a series of recent papers [7, 8, 9] authors proposed a new point of view by constructing networks exhibiting scale-free structures following ideas taken from fractal construction, e.g. Koch curve or Sierpinski gasket. The aim of the present paper is to generalize these latter constructions and to define a unifying theory, hereby named Weighted Fractal Networks, WFN for short, whose networks share with fractal sets several interesting properties, for instance the self-similarity.

The WFN are constructed via an explicit algorithm and we are able to completely analytically characterize their topology as a function of the parameters involved in the construction. We are thus able to prove that WFN exhibit the "small-world" property, i.e. slow (logarithmic) increase of the average shortest path with the network size, and large average clustering coefficient. Moreover the probability distribution of node strength follows a power law whose exponent is the Hausdorff (fractal) dimension of the "underlying" fractal, hence the WFN are scale-free.

WFN also represent an explicitely computable model for the renormalization procedure recently applied to complex networks 10, 11, 12].

The paper is organized as follows. In the next section we will introduce the model and we outline the similarities with fractal sets. In Section $\amalg$ we present the analytical characterization of such networks also supported by dedicated numerical simulations. We then introduce in Section IV a straightforward generalization of the previous theory, and thus we conclude by showing a possible application of WFN to the study of fractal structures emerging in Nature.

\section{THE MODEL}

According to Mandelbrot 13 "a fractal is by definition a set for which the Hausdorff dimension strictly exceeds the topological dimension". One of the most amazing and interesting feature of fractals is their self-similarity, namely looking at all scales we can find conformal copies of the whole set. Starting from this property one can provide rules to build up fractals as fixed point of Iterated Function Systems [14, 15], IFS for short, whose Hausdorff dimension is completely characterized by two main parameters, the number of copies $s>1$ and the scaling factor $0<f<1$ of the IFS. Let us observe that in this case this dimension coincides with the so called similarity dimension [15], $d_{\text {fract }}=-\log s / \log f$. 


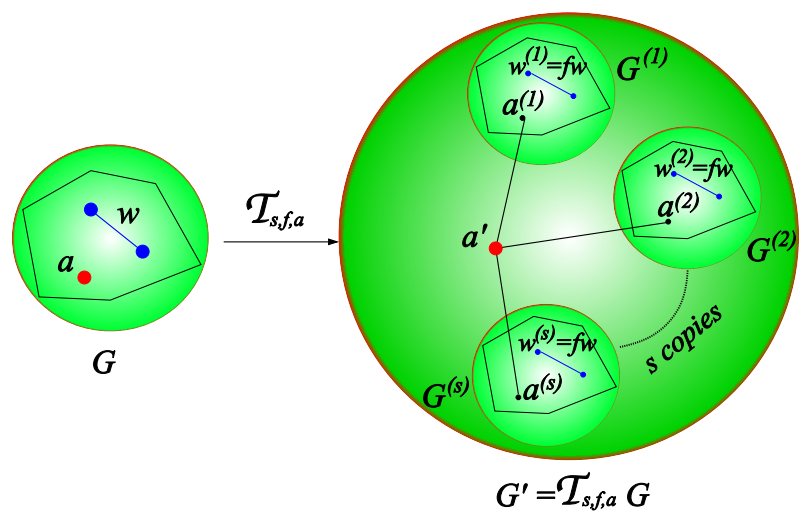

FIG. 1: The definition of the map $\mathcal{T}_{s, f, a}$. On the left a generic initial graph $G$ with its attaching node $a$ (red on-line) and a generic weighted edge $w \in G$ (blue on-line). On the right the new graph $G^{\prime}$ obtained as follows: Let $G^{(1)}, \ldots, G^{(s)}$ be $s$ copies of $G$, whose weighted edges (blue on-line) have been scaled by a factor $f$. For $i=1, \ldots, s$ let us denote by $a^{(i)}$ the node in $G^{(i)}$ image of the labeled node $a \in G$, then link all those labeled nodes to a new node $a^{\prime}$ (red on-line) through edges of unitary weight. The connected network obtained linking the $s$ copies $G^{(i)}$ to the node $a^{\prime}$ will be by definition the image of $G$ through the map: $G^{\prime}=\mathcal{T}_{s, f, a}(G)$.

The main goal of this paper is to generalize such ideas to networks, aimed at constructing weighted complex networks [20] with some a priori prescribed topology, that will be described in terms of node strength distribution, average (weighted) shortest path and average (weighted) clustering coefficient, depending on the two main parameters: the number of copies and the scaling factor [21]. Moreover taking advantage of the similarity with the IFS fractals, some topological properties of the networks will depend on the fractal dimension of the IFS fractal.

Let us fix a positive real number $f<1$ and a positive integer $s>1$ and let us consider a (possibly) weighted network $G$ composed by $N$ nodes, one of which has been labeled attaching node and denoted by $a$. We then define a map, $\mathcal{T}_{s, f, a}$, depending on the two parameters $s, f$ and on the labeled node $a$, whose action on networks is described in Fig. 1].

So starting with a given initial network $G_{0}$ we can construct a family of weighted networks $\left(G_{k}\right)_{k \geq 0}$ iteratively applying the previously defined map: $G_{k}:=\mathcal{T}_{s, f, a}\left(G_{k-1}\right)$.

Because of its general definition, the map $\mathcal{T}_{s, f, a}$ improves the constructions recently proposed in [7, 8, 9], allowing us to consider all possible IFS fractals in a unified scheme instead of using "ad hoc" constructions. For the sake of completeness we present numerical results for two WFN: the Sierpinski one (see Fig. 2) and the Cantor dust (see Fig. [3).

Given $G_{0}$ and the map $\mathcal{T}_{s, f, a}$ we are able to completely characterize the topology of each $G_{k}$ and also of the limit network $G_{\infty}$, defined as the fixed point of the map: $G_{\infty}=\mathcal{T}_{s, f, a}\left(G_{\infty}\right)$. Thus the WFN undergo through a growth process strictly related to the inverse of the renormalization procedure [10, 11]; at the same time $G_{\infty}$ will be infinitely renormalizable.

\section{RESULTS}

The aim of this section is to characterize the topology of the graphs $G_{k}$ for all $k \geq 1$ and $G_{\infty}$, by analytically studying their properties such as the average degree, the node strength distribution, the average (weighted) shortest path and the average (weighted) clustering coefficient.

At each iteration step the graph $G_{k}$ grows as the number of its nodes increases according to

$$
N_{k}=s^{k} N_{0}+\left(s^{k}-1\right) /(s-1),
$$

being $N_{0}$ the number of nodes in the initial graph, while the number of edges satisfies

$$
E_{k}=s^{k} E_{0}+s\left(s^{k}-1\right) /(s-1)
$$

being $E_{0}$ the number of edges in the graph $G_{0}$. Hence in the limit of large $k$ the average degree is finite and it is asymptotically given by

$$
\frac{E_{k}}{N_{k}} \underset{k \rightarrow \infty}{\longrightarrow} \frac{s+E_{0}(s-1)}{1+(s-1) N_{0}}
$$




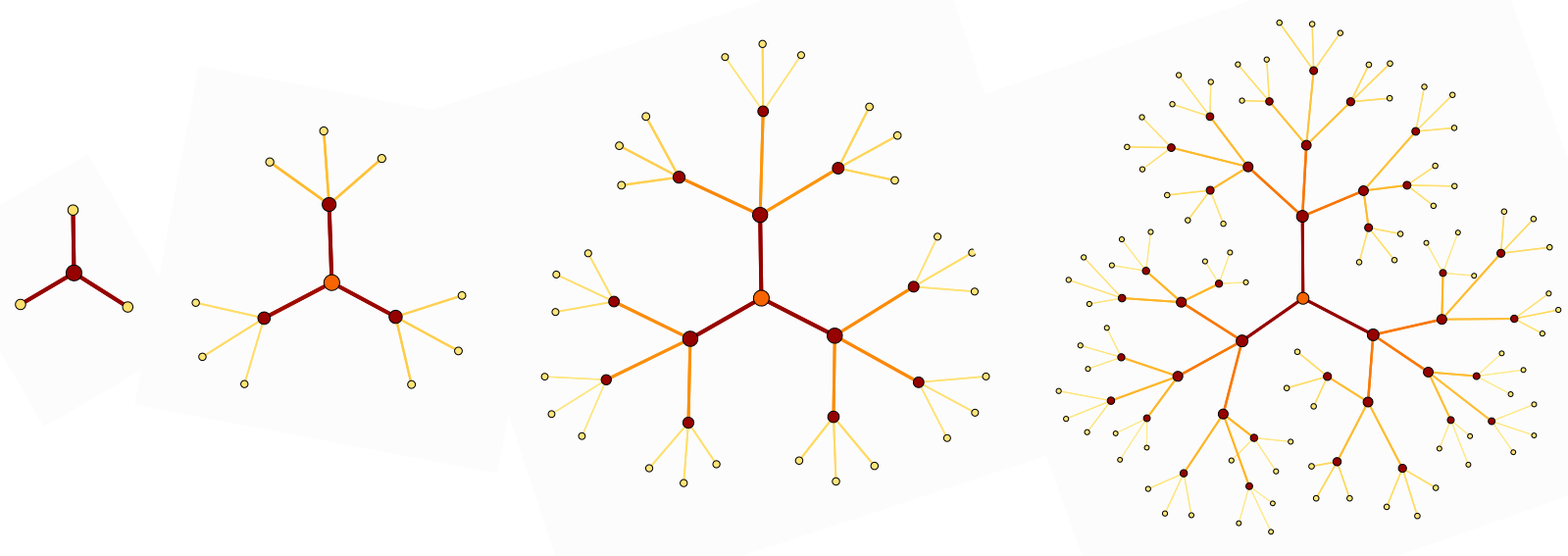

FIG. 2: The "Sierpinski" WFN, $s=3, f=1 / 2$ and $G_{0}$ is composed by a single node. From the left to the right $G_{1}, G_{2}, G_{3}$ and $G_{4}$. Gray scale (color on-line) reproduces edges weights: the darker the color the larger the weight. The dimension of the fractal is $\log 3 / \log 2 \sim 1.5850$. Visualization was done using Himmeli software [17].

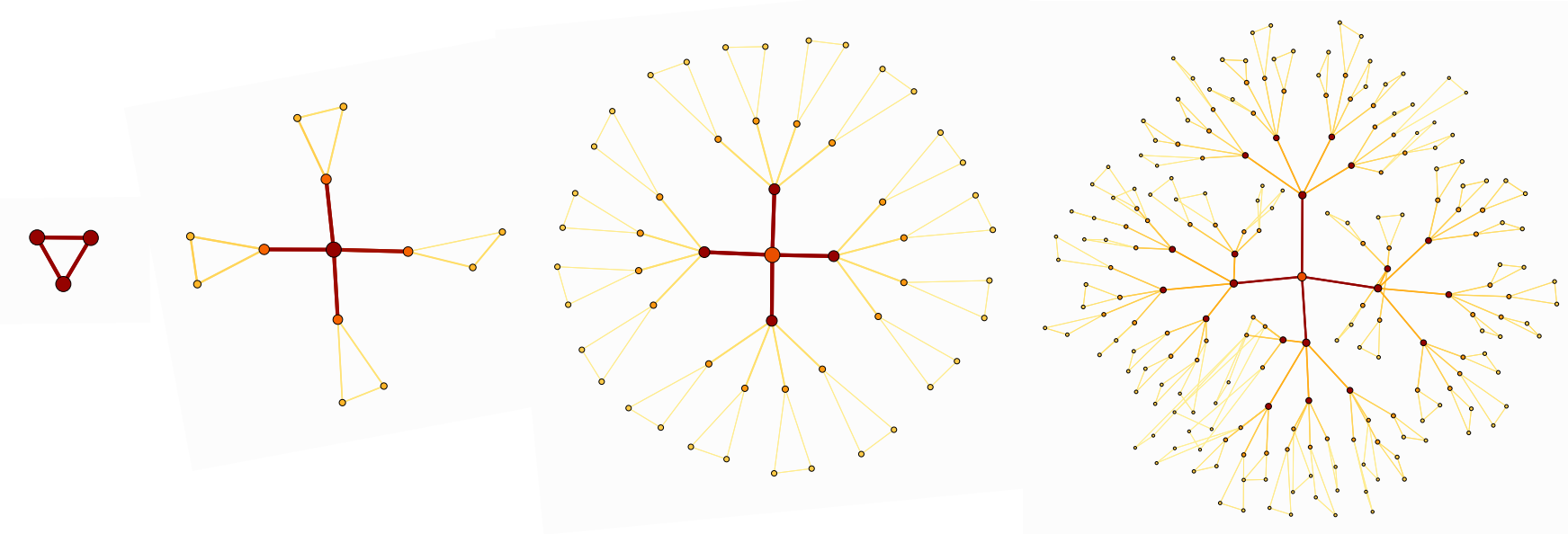

FIG. 3: The "Cantor dust" WFN, $s=4, f=1 / 5$ and $G_{0}$ is a triangle. From the left to the right $G_{0}, G_{1}, G_{2}$ and $G_{3}$. Gray scale (color on-line) reproduces edges weights: the darker the color the larger the weight. The dimension of the fractal is $\log 4 / \log 5 \sim 0.8614$. Visualization was done using Himmeli software [17].

Let us denote the weighted degree of node $i \in G_{k}$, also called node strength [18], by $\omega_{i}^{(k)}=\sum_{j} w_{i j}^{(k)}$, being $w_{i j}^{(k)}$ the weight of the edge $(i j) \in G_{k}$; then using the recursive construction, we can explicitly compute the total node strength, $W_{k}=\sum_{i} \omega_{i}^{(k)}$, and, provided $s f \neq 1$, easily show that

$$
W_{k}=2 s \frac{(s f)^{k}-1}{s f-1}+(s f)^{k} W_{0} .
$$

Because $f<1$, we trivially find that the average node strength goes to zero as $k$ increases: $W_{k} / N_{k} \underset{k \rightarrow \infty}{\longrightarrow} 0$. 


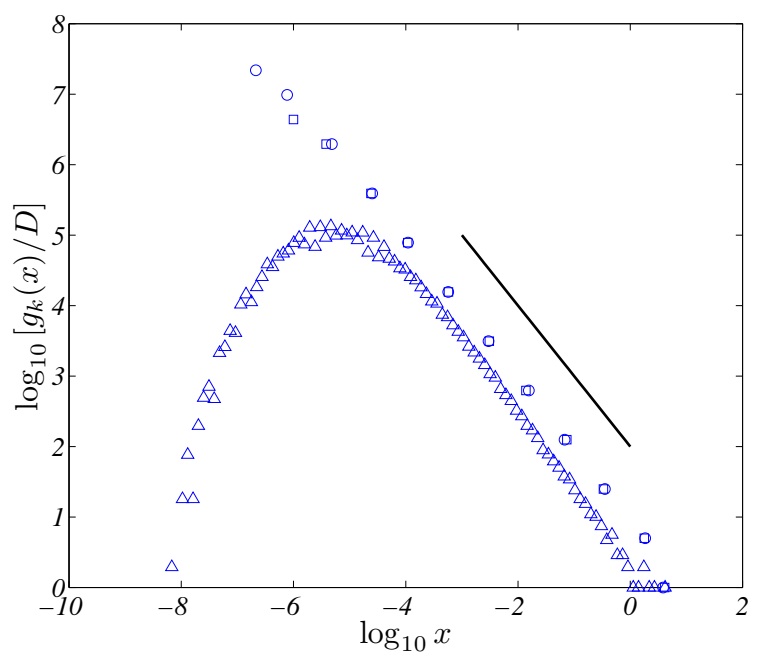

FIG. 4: Node Strengths Distribution. Plot of the renormalized node strengths distribution $\log _{10}\left[g_{k}(x) / D\right]$, where $D=d_{f r a c}$ in the homogeneous case, while $D=-\frac{s \log s}{\log \left(f_{1} \ldots f_{s}\right)}$ in the non-homogeneous one. Symbols refer to: $\square$ the finite approximation $G_{14}$ with 2391484 nodes of the "Sierpinski" WFN, $s=3, f=1 / 2$ and $G_{0}$ is formed by one initial node; $\bigcirc$ the finite approximation $G_{11}$ composed by 3495253 nodes of the "Cantor dust" WFN, $s=4, f=1 / 5$ and $G_{0}$ is made by a triangle; $\triangle$ the finite approximation $G_{11}$ composed by 3495253 nodes of the non-homogeneous "Cantor dust" WFN, $s=4, f_{1}=1 / 2, f_{2}=1 / 3$, $f_{3}=1 / 5, f_{4}=1 / 7$ and $G_{0}$ is formed by a triangle. The reference line has slope -1 ; linear best fits (data not shown) provide a slope $-0.9964 \pm 0.034$ with $R^{2}=0.9993$ for the Sierpinski WFN, a slope $-1.002 \pm 0.064$ with $R^{2}=0.9996$ for the Cantor dust WFN and a slope $-1.006 \pm 0.024$ with $R^{2}=0.9976$ for the non-homogeneous Cantor dust WFN.

\section{A. Node strength distribution.}

Let $g_{k}(x)$ denote the number of nodes in $G_{k}$ that have strength $\omega_{i}^{(k)}=x$ and let us assume $g_{0}$ to have values in some finite discrete subset of the positive reals, namely:

$$
g_{0}(x)>0 \text { if and only if } x \in\left\{x_{1}, \ldots, x_{m}\right\},
$$

otherwise $g_{0}(x)=0$. Using the property of the map $\mathcal{T}_{s, f, a}$ we straightforwardly get $g_{k}(x)=s g_{k-1}(x / f)$ provided [22] $x \neq s$ and $x \neq f s+1$, from which we can conclude that for all $k$ :

$$
g_{k}(x)=s^{k} g_{0}\left(x / f^{k}\right), \quad g_{k}(f s+1)=s \quad \text { and } \quad g_{k}(s)=1 .
$$

This implies than the node strengths are distributed according to a power law with exponent $d_{\text {fract }}=-\log s / \log f$, that equals the fractal dimension of the fractal obtained as fixed point of the IFS with the same parameters $s$ and $f$. In fact defining $x_{i k}=f^{k} x_{i}$ we get:

$$
\begin{aligned}
\log g_{k}\left(x_{i k}\right) & =k \log s+\log g_{0}\left(x_{i}\right) \\
& =\frac{\log s}{\log f} \log x_{i k}+\log g_{0}\left(x_{i}\right)-\frac{\log s}{\log f} \log x_{i},
\end{aligned}
$$

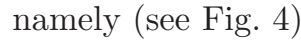

$$
g_{k}(x) \sim C / x^{d_{\text {frac }}} .
$$

\section{B. Average weighted shortest path.}

By definition the average weighted shortest path [3] of the graph $G_{k}$ is given by

$$
\lambda_{k}=\frac{\Lambda_{k}}{N_{k}\left(N_{k}-1\right)},
$$




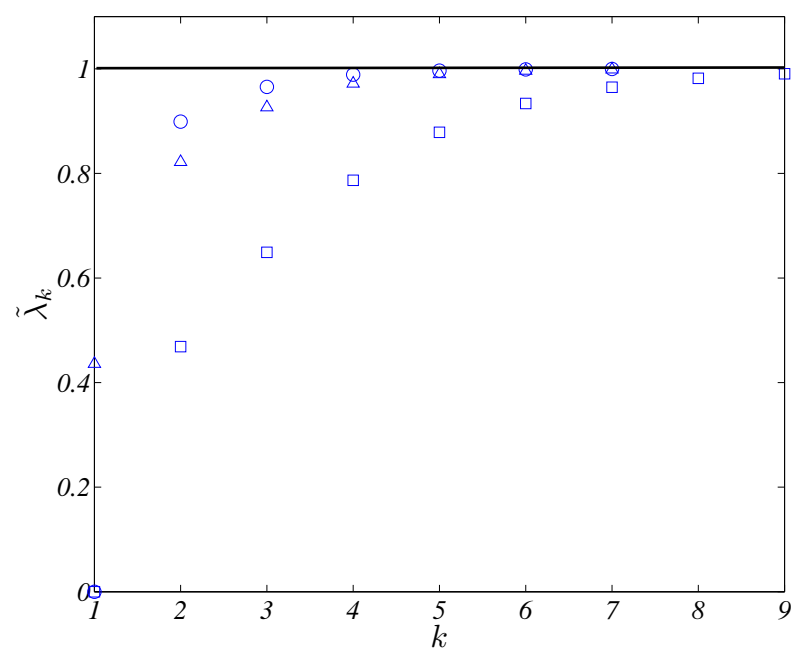

FIG. 5: The average weighted shortest path. Plot of the renormalized average weighted shortest path $\tilde{\lambda}_{k}$ versus the iteration number $k$, where $\tilde{\lambda}_{k}=\lambda_{k} \frac{(s-F)\left(s^{2}-F\right)}{2 s^{2}(s-1)}$ and $F=f_{1}+\cdots+f_{s}$ for the non-homogeneous case, while $F=s f$ for the homogeneous one. Symbols refer to : $\square$ the "Sierpinski" WFN, $s=3, f=1 / 2$ and $G_{0}$ is formed by one initial node; $\bigcirc$ the "Cantor dust" WFN, $s=4, f=1 / 5$ and $G_{0}$ is made by a triangle; $\triangle$ the non-homogeneous "Cantor dust" WFN, $s=4, f_{1}=1 / 2, f_{2}=1 / 3$, $f_{3}=1 / 5, f_{4}=1 / 7$ and $G_{0}$ is formed by a triangle.

where

$$
\Lambda_{k}=\sum_{i j \in G_{k}} p_{i j}^{(k)}
$$

being $p_{i j}^{(k)}$ the weighted shortest path linking nodes $i$ and $j$ in $G_{k}$.

To simplify the remaining part of the proof it is useful to introduce $\Lambda_{k}^{\left(a_{k}\right)}=\sum_{i \in G_{k}} p_{i a_{k}}^{(k)}$, i.e. the sum of all weighted shortest paths ending at the attaching node, $a_{k} \in G_{k}$. One can prove (see Appendix A 1) that for large $k$ the asymptotic behavior of $\Lambda_{k}^{\left(a_{k}\right)}$ is given by

$$
\Lambda_{k}^{\left(a_{k}\right)} \underset{k \rightarrow \infty}{\sim} \frac{N_{0}(s-1)+1}{(1-f)(s-1)} s^{k-1} .
$$

Using the construction algorithm and its symmetry one can prove (see again the Appendix A2) that $\Lambda_{k}$ satisfies the recursive relation

$$
\Lambda_{k}=s f \Lambda_{k-1}+2 s\left[(s-1) N_{k-1}+1\right]\left[N_{k-1}+f \Lambda_{k-1}^{\left(a_{k-1}\right)}\right],
$$

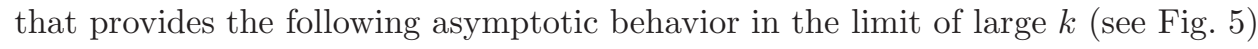

$$
\lambda_{k}=\frac{\Lambda_{k}}{N_{k}\left(N_{k}-1\right)} \underset{k \rightarrow \infty}{\longrightarrow} \frac{2(s-1)}{(1-f)(s-f)} .
$$

We can also compute the average shortest path, $\ell_{k}$, formally obtained by setting $f=1$ in the previous formulas (6) and (7). Hence slightly modifying the results previously presented we can prove that asymptotically we have

$$
\ell_{k} \underset{k \rightarrow \infty}{\sim} 2\left(k-\frac{s}{s-1}\right) \underset{k \rightarrow \infty}{\sim} \frac{2}{\log s} \log N_{k},
$$

where the last relation has been obtained using the growth law of $N_{k}$ given by equation (10) (see Fig. 66). Let us remark that the average shortest path is a topological quantity and thus it doesn't depend on the scaling factor, that's why we don't report in Fig. 6 the case of the non-homogeneous WFN.

Thus, as previously stated, the network grows unbounded but with the logarithm of the network size, while the weighted shortest distances stay bounded. 


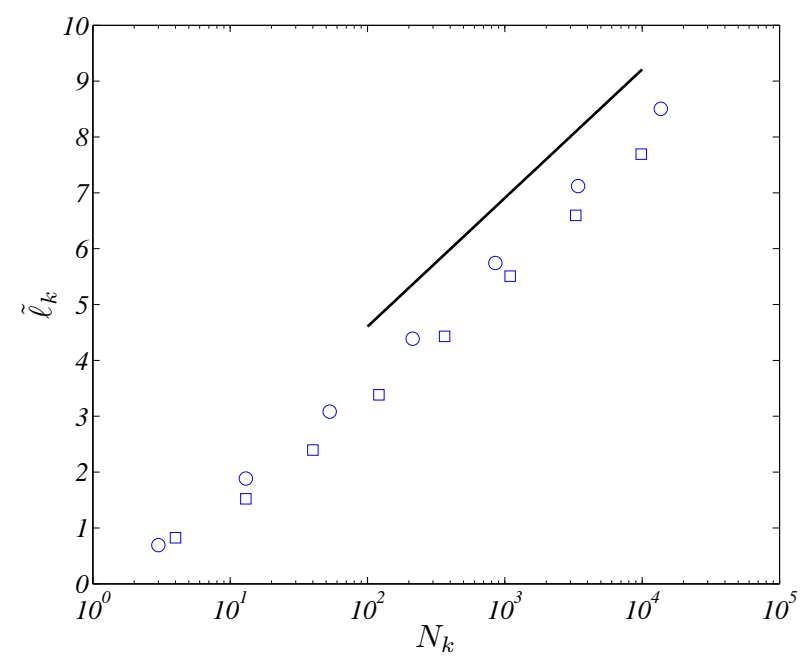

FIG. 6: The average shortest path $\ell_{k}$ as a function of the network size (semilog graph). Plot of the renormalized average shortest path $\tilde{\ell}_{k}$ versus the network size $N_{k}$, where $\tilde{\ell}_{k}=\ell_{k} \frac{\log s}{2}$. Symbols refer to : $\square$ the "Sierpinski" WFN, $s=3, f=1 / 2$ and $G_{0}$ is formed by one initial node; $\bigcirc$ the "Cantor dust" WFN, $s=4, f=1 / 5$ and $G_{0}$ is made by a triangle. The reference line has slope 1. Linear best fits (data not shown) provides a slope $0.9942 \pm 0.019$ and $R^{2}=1$ for the Sierpinski WFN and a slope $0.9952 \pm 0.019$ and $R^{2}=1$ for the Cantor dust WFN.

\section{Average clustering coefficient.}

The average clustering coefficient [3, 5] of the graph $G_{k}$ is defined as the average over the whole set of nodes of the local clustering coefficient $c_{i}^{(k)}$, namely $\left\langle c_{k}\right\rangle=C_{k} / N_{k}$, where $C_{k}=\sum_{i \in G_{k}} c_{i}^{(k)}$. Because of the construction algorithm the number of possible triangles, hence the local clustering coefficient, at each step increases by a factor $s$; thus after $k$ iterations we will have $C_{k}=s^{k} C_{0}$, being $C_{0}$ the sum of local clustering coefficients in the initial graph. We can thus conclude that the clustering coefficient of the graph is asymptotically given by:

$$
<c_{k}>\underset{k \rightarrow \infty}{\longrightarrow} \frac{s-1}{s} \frac{<c_{0}>N_{0}}{(s-1) N_{0}+1} .
$$

On the other hand, one can use edges' values to weigh the clustering coefficient [19]; hence generalizing the previous relation, we can easily prove that the average weighted clustering coefficient of the graph is asymptotically given by:

$$
<\gamma_{k}>\underset{k \rightarrow \infty}{\sim} \frac{s-1}{f s} \frac{<\gamma_{0}>N_{0}}{(s-1) N_{0}+1} f^{k} \underset{k \rightarrow \infty}{\sim} \frac{1}{N_{k}^{1 / d_{\text {fract }}}},
$$

where once again, the fractal dimension $d_{\text {fract }}$ of the IFS fractal play a relevant role.

\section{NON-HOMOGENEOUS WEIGHTED FRACTAL NETWORKS}

The aim of this section is to slightly generalize the previous construction to the case of non-homogeneous scaling factors for each subnetwork $G^{(i)}$. So given an integer $s>1$ and $s$ real numbers $f_{1}, \ldots, f_{s} \in(0,1)$, we modify the map $\mathcal{T}_{s, f, a}$ by allowing a different scaling for each edge weight according to which subgraph it belongs to: if the edge $w^{(j)}$, image of $w \in G$, belongs to $G^{(j)}$, then $w^{(j)}=f_{j} w$.

Let us remark that the construction presented in the former Section 1 is a particular case of the latter once we take $f_{1}=\cdots=f_{s}=f$; we nevertheless decided for a sake of clarity, to present it before, because the computations involved in this latter general construction could have hidden the simplicity of the underlying idea. We hereby present some results for the non-homogeneous "Cantor dust"WFN (see Fig. (7).

Using the recursiveness of the algorithm we can, once again, completely characterize the topology of the nonhomogeneous WFN, moreover only the weighted quantities will vary with respect to the homogeneous case. For instance, a straightforward, but cumbersome, generalization of the computations presented in the previous Sections 

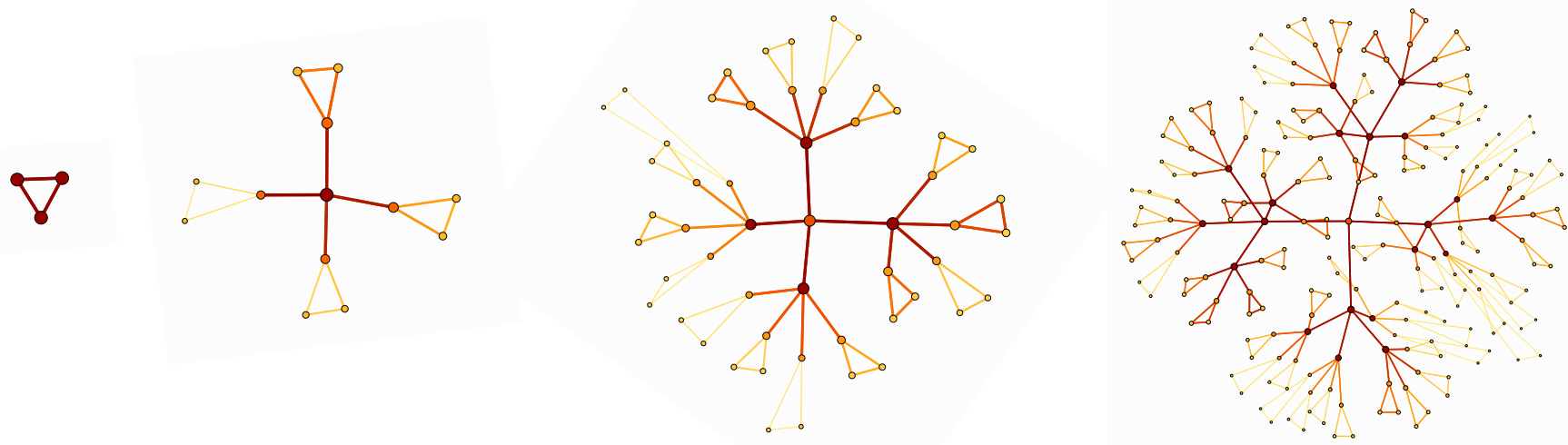

FIG. 7: The non-homogeneous "Cantor dust" WFN, $s=4, f_{1}=1 / 2, f_{2}=1 / 3, f_{3}=1 / 5, f_{4}=1 / 7$ and $G_{0}$ is formed by a triangle. From the left to the right $G_{0}, G_{1}, G_{2}$ and $G_{3}$. Gray scale (color on-line) reproduces edges weights: the darker the color the larger the weight. Visualization was done using Himmeli software 17].

allows us to prove that the average weighted shortest path exhibits the following asymptotic behavior (see Fig. [5)

$$
\lambda_{k} \underset{k \rightarrow \infty}{\longrightarrow} \frac{2 s^{2}(s-1)}{(s-F)\left(s^{2}-F\right)},
$$

where $F=f_{1}+\cdots+f_{s}$. Let us observe that Eq. (14) reduces to Eq. (10) once we set $f_{1}=\cdots=f_{s}=f$ and thus $F=s f$.

Let $g_{0}(x)$ denote the number of nodes with node strength equal to $x$ in the initial network $G_{0}$; then after $k$ steps of the algorithm, all nodes strengths will be rescaled by a factor $f_{1}^{k_{1}} \ldots f_{s}^{k_{s}}$, where the non-negative integers $k_{i}$ do satisfy $k_{1}+\cdots+k_{s}=k$. Because this can be done in $k ! /\left(k_{1} ! \ldots k_{s} !\right)$ possible different ways, we get the following relation for the node strength distribution for the network $G_{k}$ :

$$
g_{k}\left(f_{1}^{k_{1}} \ldots f_{s}^{k_{s}} x\right)=\frac{k !}{k_{1} ! \ldots k_{s} !} g_{0}(x) \quad \text { with } k_{1}+\cdots+k_{s}=k .
$$

After sufficiently many steps and assuming that the main contribution arises from the choice $k_{1} \sim \cdots \sim k_{s} \sim k / s$, we can use Stirling formula to get the approximate distribution (see Fig. 4)

$$
\log g_{k}(x) \sim \frac{s \log s}{\log \left(f_{1} \ldots f_{s}\right)} \log x,
$$

so once again the nodes strength distribution follows a power law.

\section{CONCLUSIONS}

In this paper we introduced a unifying framework for complex networks sharing several properties with fractal sets, hereby named Weighted Fractal Networks. This theory, that generalizes to graphs the construction of IFS fractals, allows us to build complex networks with a prescribed topology, whose main quantities can be analytically predicted and have been shown to depend on the fractal dimension of the IFS fractal; for instance the networks are scale-free with exponent the fractal dimension. Moreover the weighted fractal networks share with IFS fractals, the self-similarity structure, and are explicitely computable examples of renormalizable complex networks.

These networks exhibit the small-world property. In fact the average shortest path increases logarithmically with the system size (11), hence it is small as the average shortest path of a random network with the same number of nodes and same average degree. On the other hand the clustering coefficient is asymptotically constant (12), thus larger than the clustering coefficient of a random network that shrinks to zero as the system size increases. 
The self-similarity property of the weighted fractal networks makes them suitable to model real problems involving generic diffusion over the network coupled with local looses of flow, here modeled via the parameter $f<1$. For instance one can think of electrical grids or mammalian lungs, where current or air, flows through power lines or bronchi-bronchioles, submitted to looses of power, or air vessels' section reduction. In all these cases the induced topology, namely a good choice of $f$ and $s$, allows any two random nodes, final power users or alveoli, to be always at finite weighted distance, whatever their physical distance is, and thus to be able to transport current or oxygen in finite time.

\section{APPENDIX A: COMPLEMENTARY MATERIAL}

\section{Computation of $\Lambda_{k}^{\left(a_{k}\right)}$}

Let $a_{k}$ be the attaching node of the graph $G_{k}$. Let us define $\Lambda_{k}^{\left(a_{k}\right)}=\sum_{i \in G_{k}} p_{i a_{k}}^{(k)}$, i.e. the sum of all weighted shortest paths to $a_{k}$. Then using the recursive property and the symmetry of the map $\mathcal{T}_{s, f, a_{k}}$ we can easily obtain a recursive relation for $\Lambda_{k}^{\left(a_{k}\right)}$ :

$$
\Lambda_{k}^{\left(a_{k}\right)}=s f \Lambda_{k-1}^{\left(a_{k-1}\right)}+s N_{k-1}
$$

where $N_{k-1}$ is the number of nodes in $G_{k-1}$. This recursion can be easily solved to get for all $k \geq 1$

$$
\Lambda_{k}^{\left(a_{k}\right)}=(s f)^{k-1} \Lambda_{1}^{\left(a_{1}\right)}+\frac{1-f^{k-1}}{1-f} \frac{(s-1) N_{0}+1}{s-1} s^{k-1}-\frac{s}{s-1} \frac{(s f)^{k-1}-1}{s f-1},
$$

from which we can conclude, because $f<1$, that $\Lambda_{k}^{\left(a_{k}\right)}$ exhibits the asymptotic behavior given by equation (8).

\section{Computation of $\Lambda_{k}$}

Starting from the definition of the sum of all weighted shortest paths (7), the recursive construction and its symmetry we can decompose the sum $\Lambda_{k}$ into three terms:

$$
\Lambda_{k}=s \sum_{i j \in G_{k}^{(1)}} p_{i j}^{(k)}+s(s-1) \sum_{i \in G_{k}^{(1)}, j \in G_{k}^{(2)}} p_{i j}^{(k)}+2 s \sum_{i \in G_{k}^{(1)}} p_{i a_{k}}^{(k)}
$$

where the first contribution takes into account all paths starting from and arriving to nodes belonging to the same subgraph, that using the symmetry can be chosen to be $G_{k}^{(1)}$. The second term takes into account all the possible paths where the initial point and the final one belong to two different subgraphs, and still using the symmetry we can set them to $G_{k}^{(1)}$ and $G_{k}^{(2)}$ and multiply the contribution by a combinatorial factor $s(s-1)$. Finally the last term is the sum of all paths arriving to the attaching node $a_{k}$; once again the symmetry allows us to reduce the sum to only one subgraph, say $G_{k}^{(1)}$, and multiply the contribution by $2 s$.

Using the scaling mechanism for the edges, the first term in the right hand side of equation (A2) can be easily identified with

$$
\sum_{i j \in G_{k}^{(1)}} p_{i j}^{(k)}=f \Lambda_{k-1}
$$

By construction, each shortest path connecting two nodes belonging to two different subgraphs, must pass through the attaching node, hence using $p_{i j}^{(k)}=p_{i a_{k}}^{(k)}+p_{a_{k} j}^{(k)}$ the second term of equation (A2) can be split into two parts:

$$
\sum_{i \in G_{k}^{(1)}, j \in G_{k}^{(2)}} p_{i j}^{(k)}=\sum_{i \in G_{k}^{(1)}} p_{i a_{k}}^{(k)} N_{k}^{(2)}+\sum_{j \in G_{k}^{(2)}} p_{a_{k} j}^{(k)} N_{k}^{(1)}
$$

where $N_{k}^{(i)}$ denotes the number of nodes in the subgraph $G_{k}^{(i)}$. Using the symmetry of the construction, the previous relation can be rewritten as

$$
\sum_{i \in G_{k}^{(1)}, j \in G_{k}^{(2)}} p_{i j}^{(k)}=2 N_{k}^{(1)} \sum_{i \in G_{k}^{(1)}} p_{i a_{k}}^{(k)}
$$


The last term of equation (A2) can be related to $\Lambda_{k-1}^{\left(a_{k-1}\right)}$ by observing that each path arriving at $a_{k}$ must pass through $a_{k}^{(i)}$ for some $i \in\{1, \ldots, s\}$, thus

$$
\begin{aligned}
\sum_{i \in G_{k}^{(1)}} p_{i a_{k}}^{(k)} & =\sum_{i \in G_{k}^{(1)}}\left(p_{i a_{k}^{(1)}}^{(k)}+p_{a_{k}^{(1)} a_{k}}^{(k)}\right)=N_{k}^{(1)}+\sum_{i \in G_{k}^{(1)}} p_{i a_{k}^{(1)}}^{(k)} \\
& =N_{k}^{(1)}+f \Lambda_{k-1}^{\left(a_{k-1}\right)},
\end{aligned}
$$

Observing that $G_{k}^{(1)}$ has as many nodes as $G_{k-1}$ we can conclude that $N_{k}^{(1)}=N_{k-1}$ and finally to rewrite equation (A2) as:

$$
\Lambda_{k}=s f \Lambda_{k-1}+2 s\left[(s-1) N_{k-1}+1\right]\left[N_{k-1}+f \Lambda_{k-1}^{\left(a_{k-1}\right)}\right]
$$

[1] Erdős, P. and Rényi, A. On random graphs, Publ. Math. Debrecen 6, 290, (1959).

[2] Albert, R. and Barabási, A.-L. Statistical mechanics of complex networks, Rev. Mod. Phys. 74, 47, (2002).

[3] Boccaletti, S., Latora, V., Moreno, Y., Chavez, M. and Hwang, D.-H. Complex networks: Structure and dynamics, Phys. Rep. 424, 175, (2006).

[4] Barabási, A.-L. and Albert, R. Emergence of Scaling in Random Networks, Science 286, 509, (1999).

[5] Watts, D.J. and Strogatz, S.H. Collective dynamics of 'small-world' networks, Nature 393, 440, (1998).

[6] Fortunato, S. Community detection in graphs, preprint arXiv:0906.0612 v1 [physics.soc-ph], (2009).

[7] Zhang, Z., Guan, J., Chen, L., Yin, M. and Zhou, S. Novel scale-free small-world networks from Koch curves, preprint cond-mat.stat-mech/0810.3313v1, (2008).

[8] Zhang, Z., Zhou, S., Su, Z., Zou, T. and Guan, J. Random Sierpinski network with scale-free small-world and modular structure, Eur. Phys. J. B. 65, 141-147, (2008).

[9] Guan, J., Wu, Y., Zhang, Z., Zhou, S. and Wu, Y. A unified model for Sierpinski networks with scale-free scaling and small-world effect, Physica A 388, 2571-2578, (2009).

[10] Song, C., Havlin, S. and Makse, H.A. Self-similarity of complex networks, Nature 433, 392, (2005).

[11] Song, C., Havlin, S. and Makse, H.A. Origin of fractality in the growth of complex networks, Nature Phys. 2, 275, (2006).

[12] Radicchi, F., Barrat, A., Fortunato, S. and Ramasco, J.J. Renormalization flows in complex networks, preprint arxiv:/physics.soc-ph/0811.2761v1, (2008).

[13] Mandelbrot, B.B. The Fractal Geometry of Nature, W.H. Freeman and Company, New York (1982).

[14] Barnsley, M. Fractals everywhere, Academic Press London (1988).

[15] Edgar, G.A. Measure, Topology and fractal geometry, UTM, Springer-Verlag, New York (1990).

[16] Carletti, T. Stochastic Weighted Fractal Networks, preprint (2009).

[17] Mäkinen, V. Himmeli, a free software package for visualizing complex networks, available at http://www.artemis.kll.helsinki.fi/himmeli

[18] Barrat, A., Barthélemy, M., Pastor-Satorras, R. and Vespignani, A. The architecture of complex weighted networks, Proc. Natl. Acad. Sci. USA 101, 3747, (2004).

[19] Saramäki, J., Kivelä, M., Onnela, J.-P., Kaski, K. and Kertész, J. Generalizations of the clustering coefficient to weighted complex networks, Phys. Rev. E 75, 027105, (2007).

[20] We hereby present the construction for undirected networks, but it can be straightforwardly generalized to directed graphs as well.

[21] A straightforward generalization will be presented in the next Section IV] See also [16] where the WFN theory will be generalized as to include a stochastic iteration process.

[22] Without loose of generality we can assume that for all integers $i, j \in\{1, \ldots, m\}$ and $k>0$ we have $f^{k} x_{j} \neq x_{i}$ and $f^{k}\left(f x_{j}+1\right) \neq x_{i}$. 